\title{
Assessing U.S. Travelers' Trade-offs for Aviation Safety Objectives: A Natural Experiment
}

\author{
Kenneth Nguyen \\ University of Southern \\ California \\ hoangdun@usc.edu
}

\author{
Heather Rosoff \\ University of Southern \\ California \\ rosoff@usc.edu
}

\author{
Richard S. John \\ University of Southern \\ California \\ richardj@usc.edu
}

\begin{abstract}
Understanding air travelers' values for aviation safety is essential to design effective and wellaccepted security measures. This study investigates changes in U.S. travelers trade-offs for passenger screening objectives using the occurrence of an international aviation incident (loss of Malaysian Airline Flight 370) as a natural experiment. We also examine how alternative screening procedures affect trade-offs between equity and safety concerns. Results show evidence for an enduring effect of the aviation incident on trade-offs between safety and other passenger screening objectives. Additionally, the use of different procedures to select high-risk passengers for enhanced screening altered the relative importance of the equity objective. Implications for the design of future airport security policies are discussed.
\end{abstract}

\section{Introduction}

Due to heightened public concern over aviation safety ${ }^{[1]}$, the Transportation Security Administration (TSA) has proposed and implemented a variety of new security procedures at U.S. airports in recent years. Whereas numerous initiatives have been proposed to make air travel safer, creating an effective and well-accepted airport security policy has been a challenge for the TSA. This is because the evolution of U.S. passenger screening technologies and policies has resulted in increasing passenger concerns related to conflicting objectives. Employing a procedure that maximizes safety will undoubtedly increase cost, wait time, and inconvenience. Importantly, some of the proposed security measures may be perceived to specifically target passengers with characteristics related to known members of terrorist organizations. However, the classification of high versus low risk passengers for additional security scrutiny raises the issue of unequal treatment of passengers of different nationality, ethnicity, race, sex, age, or religion.
As aviation safety will continue being an ongoing public concern, new policies that aim to increase safety while enhancing efficiency and maintaining public acceptance should take into account U.S. travelers' personal values and willingness to accept sacrifices to make air travel safer. Importantly, tradeoffs for safety are likely to evolve in response to terrorist threats and screening vulnerabilities. It is unlikely that people constantly worry about air travel when there has been no incident that highlights risks of air travel. Conversely, travelers will be more likely to make greater sacrifices for safety when there is a direct threat applied to air travel. Surprisingly, there has been no study that directly addresses how tradeoffs change over time. The current study addresses this lacuna by modeling trade-offs among conflicting objectives for passenger screening in commercial aviation. In particular, we utilized the disappearance of Malaysian Airline Flight 370 to model changes in trade-offs for safety over time.

Because previous policies that aim to increase public safety have been criticized on the grounds that they violate the constitutional principal of equal protection ${ }^{2]}$, equity appears to be another important value that many U.S. travelers wish to protect. However, policies that aim to increase safety may jeopardize this principle, requiring travelers to tradeoff safety and equity objectives. In addition, different security procedures may be interpreted quite differently with respect to equity values. For example, a randomized search at checkpoint appears to be more equitable than a search based on profiling based on individual passenger characteristics such as nationality, ethnicity, race, sex, age, or religion. Thus, how passengers are selected for greater scrutiny may also affect the trade-off between equity and safety. A secondary aim of this research is to explore whether and how different types of selection procedures for passenger screening affect equity trade-offs. 


\section{Trade-off between Safety and Equity}

\subsection{Travelers' Multi-Attribute Utility Model}

U.S. travelers have multiple concerns and priorities in relation to changing passenger screening policies. Although concern for safety is central, equity, time, cost, and convenience are also top priorities. For instance, a survey of the airline industry indicated that removing shoes during security screening is second only to high ticket price in passenger complaints ${ }^{[3]}$. However, because a policy that helps to increase safety is likely to require sacrifices on other objectives, any policy that aims to increase aviation safety will require travelers to make trade-offs. Importantly, travelers will sacrifice some level of equity because of the use of risk-based screening in which travelers classified as high risk will be selected for a more thorough security check. The selection not only creates additional inconvenience for some travelers but also agitates others because travelers may perceive such selection as being an instance of the violation of the constitution principle of equal protection. These undesirable consequences can undermine public acceptance and confidence in the system, leading to possible withdrawal of highly effective security procedures.

Multi-Attribute Utility Theory (MAUT) [4] provides an appropriate framework to quantify travelers' trade-offs among conflicting objectives, including safety, in the context of airport security. MAUT prescribes a model to compare alternative screening policies that accounts for travelers' concern for multiple conflicting objectives. Given U.S. passenger diversity, one would expect individual differences in multi-attribute utility models among individual travelers. We apply MAUT to characterize and quantify the trade-off between safety and four other conflicting objectives that are of concern to U.S. travelers. The mathematical expression of the additive model is $\mathrm{U}\left(\mathrm{X}_{1}, \mathrm{X}_{2, \ldots}, \mathrm{X}_{\mathrm{n}}\right)=\sum_{i=1}^{n} w_{i} u_{i}\left(x_{i}\right)$ where " $U$ " is the multi-attribute utility of the passenger screening policy to the traveler; the capital " $\mathrm{X}_{\mathrm{i}}$ " represents the measurable attribute of an objective; note that multiple $\mathrm{X}_{\mathrm{i}}$ 's represent multiple (conflicting) attributes; the lower case " $\mathrm{x}_{\mathrm{i}}$ " indicates the levels of the respective attributes; the " $u_{i}$ " represents the utility functions of each attribute; and " $\mathrm{W}_{\mathrm{i}}$ " are scaling constants. The degree that an individual passenger is concerned about safety is characterized by a scaling constant " $\mathrm{W}_{\mathrm{i}}$ " with respect to the objective " $\mathrm{X}_{\mathrm{i}}$ "- "Maximizing Safety". Because the values of the scaling constants sum to 1.0, the scaling constant for the objective
"Maximizing Safety" is the relative weight of safety in the model. This value depends in part on the ranges of the measureable attributes representing the objectives. In the present study, scaling constants (weights) are assessed from individual pairwise choices varying in safety and each of the other objectives specified in the model.

In the context of airport security, we identified five relevant objectives that travelers are likely to care about. These five objectives are: maximizing equity, minimizing wait time, maximizing safety, maximizing convenience, and minimizing cost. The first three objectives are adopted from Viscusi and Zeckhauser ${ }^{[5]}$ whereas the latter two are included for completeness. Importantly, these objectives are conflicting with each other in the sense that an increase in the level of achievement in one objective generally requires a decrease in the achievement in the other objectives. For instance, a safer security procedure that requires more stringent screening and security examination undoubtedly increases the time it takes travelers to complete their security check, and increases the cost and inconvenience. Likewise, a safer screening procedure may compromise the principle of equal protection. In our conceptualization, a risk-based procedure that identifies and selects high-risk passengers for additional security check is safer than a procedure that screens passengers identically. Clearly, security resources are limited, and the majority of passengers have a very low security risk. Thus, allocating scarce resources to low risk passengers is unwise, and concentrating more resources on high-risk passengers is a more effective approach. Consequently, a safer security screening may violate the principle of equal protection because only a subset of passengers suffer the consequence of enhanced screening. Passengers may not have equal chances of selection for enhanced screening, thus creating a conflict that requires a trade-off between safety and equity.

To elicit trade-offs involving safety and the other four objectives, we designed a hypothetical decision dilemma in which respondents were asked to make binary choices requiring a trade-off for a high level of safety by giving up some level of achievement in other objectives, including cost, time, convenience, and equity. Specifically, respondents choose between two airlines for a vacation flight. The two airlines are identical in all aspects, including 3 of the 5 attributes, but they differ specifically on exactly two of the five attributes, thus requiring respondents to make a trade-off between these two attributes. Using this decision dilemma, we elicited an estimate of how much travelers were willing to sacrifice in terms of equity, money, time, and convenience to 
achieve a higher level of safety. The trade-offs for safety are referred to as safety premiums to reflect the additional costs that travelers are willing to incur to make air travel safer. From the elicited safety premiums, we compute scaling parameters (weights) for safety in the MAU model. We define the concept safety weight as the relative importance of safety with respect to our MAU model.

\subsection{Changes in Safety Scaling Parameters}

Because the safety premiums are contingent upon how travelers prioritize safety with respect to other attributes, such as time, money, equity, and convenience, it is expected that the relative weight for safety changes in response to commercial aviation events, such as accidents or terror attacks. Indeed, it is expected that the weight for equal protection is weaker when concern for safety is high compared to when it is low ${ }^{[5,6]}$. The current research attempts to directly address this prediction by using an actual aviation incident to conduct a natural longitudinal experiment to examine how preference for safety and equity change over time. We utilize the occurrence of the disappearance of the Malaysian Flight 370 to study whether and how the relative importance of safety changes as the incident unfolds.

The Malaysia Airlines Flight 370 (MH370) was a scheduled international flight from Kuala Lumpur, Malaysia to Beijing, China on March 8th 2014. The flight disappeared less than an hour after taking off from Kuala Lumpur, carrying 227 passengers from 15 countries. The disappearance of the aircraft has launched a multinational search, but after over 2 years of extensive search, the aircraft is still missing. All crew members and passengers have been presumed dead. The MH370 disappearance has resulted in extensive media coverage. During the search and investigation, several causes of the disappearance have been proposed but none has been confirmed. As of today, MH370 is still missing. The disappearance of MH 370 presents a rare and unique opportunity to examine safety trade-offs. The current study utilizes this incident to examine how U.S. travelers make trade-offs for safety over a period of six weeks after the disappearance of the plane. Every week during the 6-week study period, we invited a different sample of U.S. travelers to participate in our experiment. Figure 1 presents the timeline of the event and the points in time that we collected data.

There are two opposing hypotheses about the pattern of trade-off for safety over time. Previous research on risk perception in transportation accidents suggests a time decay hypothesis ${ }^{[7,8]}$. The prediction is that travelers are willing to make greater sacrifices for safety immediately after the incident because of the increased perception of risk to aviation safety and security, but the effect is attenuated as the incident fades. Several empirical studies provide support for this prediction. For example, Viscusi and Zeckhauser (2003) who found that compared to pre$9 / 11$, respondents were more willing to compromise their preference for equal protection to achieve a higher level of airport safety after 9/11. In addition, Sheu ${ }^{[7]}$ found that students who attended a college in an island off of mainland Taiwan in which air flights and ferries are the only means of transportation between the island the mainland showed an increased concern over safety in the two weeks after a plane crash, and a greater likelihood of traveling by ferry despite its greater travel time and inconvenience. Additional evidence comes from studies on the Tokyo anthrax attack and the Madrid bombing. Although both events resulted in civilian deaths (12 in the subway anthrax attack and 191 in the bombing), the reduction in public transportation ridership, presumably due to the fear of additional attacks, was temporary, disappearing shortly after the events ${ }^{[9,10]}$.

Conversely it is entirely possible that the perception of risk lingers over time, causing travelers to continuously worry about their travel safety due to intense media coverage of the event and the surrounding uncertainty. This effect can be further exacerbated as terrorism remains a possible cause for the incident, since the investigation has not concluded. Following this logic, safety weights are expected to stay high for an extended period of time. Support for this possibility comes from a study by Prager and colleagues ${ }^{[11]}$ who showed that there was a significant drop in subway ridership in London after the bombing attacks that killed 39 people. The reduction in ridership persisted for months, and there was evidence showing that the reduction may have continued for almost a year. In a recent study on risk perception after the collision between two Washington DC's Metrorail trains, Wernstedt and Murray-Tuite ${ }^{[12]}$ found evidence contradicting the time decay hypothesis. In particular, they found a positive and statistically significant relationship between the passage of time and safety concerns.

Given these contradictory predictions and the uniqueness of the MH370 incident, we explore both hypotheses. Because the weights in the MAU model are dependent and constrained to sum to 1.0, an increase in safety weight implies a decrease in the weight for at least one other objective. Thus, we also explore how weights for other attributes, including time, money, convenience, and equity, change over time. 


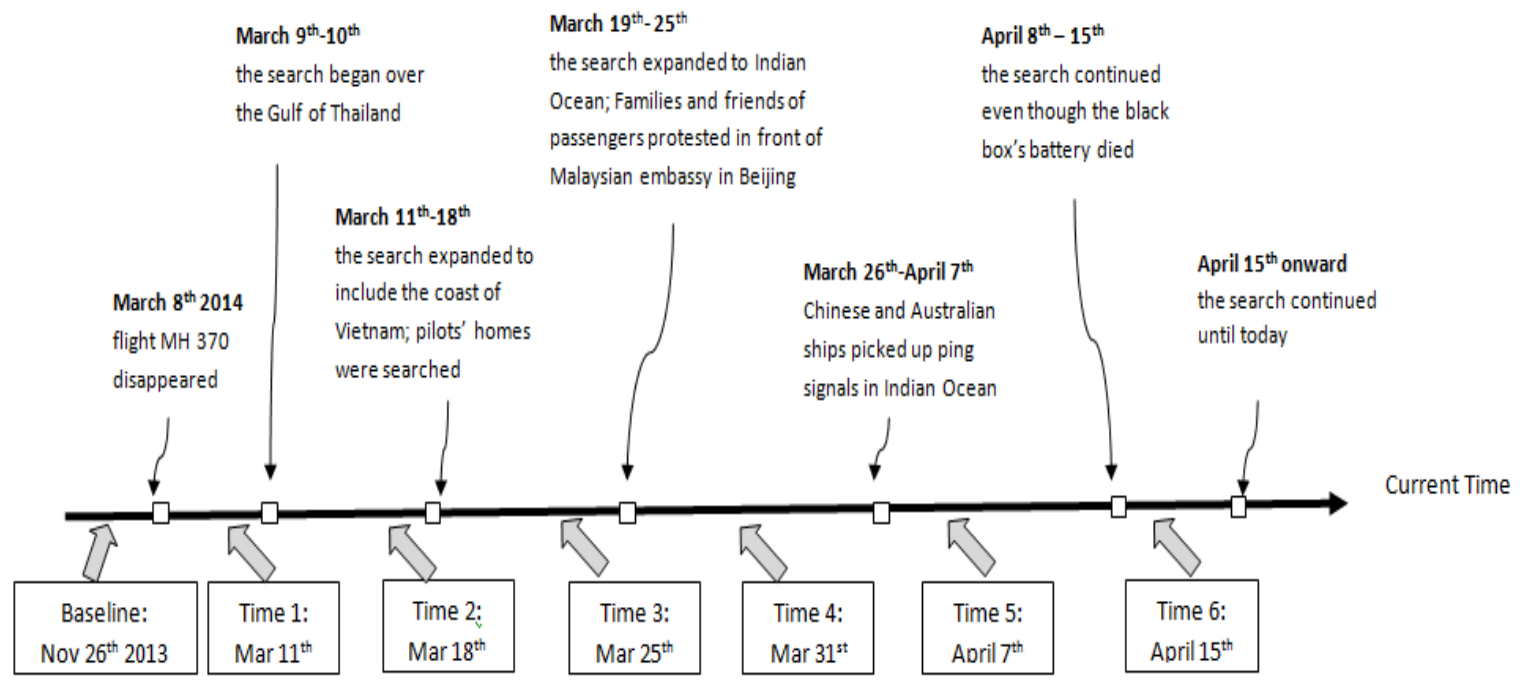

Figure 1. Timeline of the MH370 disappearance and data collection prior to and after the disappearance.

\subsection{Concern for Equity is Malleable}

Because previous airport security policies have been rejected on the grounds that they violate the constitutional principle of equal protection, understanding preference for equity offers valuable insights into the design and implementation of new policies that aim to increase aviation safety and airport security. Indeed, a particularly interesting question is how different screening alternatives may affect preference for equity. The question is motivated by the fact that the TSA has experimented with various screening procedures. The criminology literature suggests that public support for legal authorities is conditional on the perception of the fairness of the procedure by which a legal measure is implemented - a concept known as procedural justice [13]. In the airport security context, the notion of procedural justice suggests that public support for a selective security screening policy may largely depend on the characteristics of the procedure that selects passengers for additional scrutiny. In other words, the notion of procedural justice implies that equity values depend on perceptions of procedural fairness in passenger screening measures implemented by the TSA. When a fair procedure is used and is perceived as such, travelers may not have to compromise their safety priority to accommodate their preference for an equitable screening. However, when an unfair procedure is used, travelers are expected to make greater trade-offs to protect their preference for equity, potentially reducing their safety priority.
Various security selection procedures have potential implications for the trade-offs involving equity. For example, although the explicit act of profiling passengers based on race, ethnicity, age, and sex is not permitted by law, the use of profiling manifests in many facets of past and current security programs. Individuals may be selected for particular high risk lists based on personal characteristics and history that may involve protected classes and invoke the possibility of profiling. Behavioral screening is another option with unknown efficacy. The rationale behind this technique is that potential terrorists who disguise themselves as regular air travelers may express certain emotional responses such as anxiety, fear, and distress. Through extensive training, behavioral detection officers are believed to be able to detect micro changes in the facial expression of these potential terrorists, and apprehend these suspects before they can execute their action plans. A third alternative is randomized security screening. A limited version of randomized screening has been implemented for passengers who currently participate in the Trusted Travel Program. Recall that passengers who sign up for this program undergo an expedited screening. However, TSA officers still randomly select some of these travelers for additional screening.

Clearly, even though these procedures result in the same outcome such that some individuals are singled out for additional scrutiny more frequently than others, they have unique procedural characteristics that distinguish them from each other. The possible differences in the procedural characteristics can constitute unique judgmental and behavioral reactions to each of these procedures; 
hence, trade-offs for equity are possibly very different under different procedures. Surprisingly, this empirical research question has largely remained unexamined. The current study is designed to address this research gap by comparing how different selective screening procedures can influence tradeoffs involving equity. In particular, we expect that equity receives more weight under the profilingbased selection procedure and under the behavioral selection procedure compared to the randomized selection. We hypothesize that the former two procedures result in greater concern for equal protection due to subjective perceptions of fairness. While random selection necessarily results in outcome inequity, we expect travelers to perceive random screening high in procedural fairness.

\section{Method}

\subsection{Study Overview}

In November 2013, we conducted the first experiment to examine preference for equity in the context of airport security, several months prior to the MH370 incident. On March $8^{\text {th }} 2014$ news about the disappearance of the MH370 first emerged, and we began collecting additional data to examine how preference for safety changed after the occurrence of a safety-related aviation incident. The design, materials, and procedure were the same for the preand post-MH370 studies. Thus, data collected prior to the MH370 disappearance serve as the baseline measure, and data collected after March $8^{\text {th }} 2014$ allowed us to examine how relative weights for safety changed over time. During each of the next six consecutive weeks, we recruited a different sample to participate in our study (see Figure 1 for the timeline). Thus, the study is a cross-sectional longitudinal natural experiment (see Figure 1 for the timeline). All respondents are Mechanical Turk workers. Previous studies have shown that Turk samples are generally more representative than other non-probability samples [14, 15, 16]. Demographic information, including sex, age, self-reported political preference, and frequency of flight for each panel is presented in Table 1.

Each experiment began with a four-minute video describing the study, including a detailed explanation of the five objectives, as well as a careful description of the task they were going to complete. Respondents were randomly assigned to one of three possible selection procedures for the two-stage screening, i.e., randomization, behavioral indicators, or demographic profiling. Respondents completed ten pairwise tradeoff assessments derived from selecting all possible pairs of the five objectives. The order of the trade-off assessments was randomized. In each of the assessments, respondents were offered two alternative screening policies (varying on two attributes only) and were asked to indicate their preferred option or indifference.

\subsection{Objective Definitions}

The five objectives specified in our MAU model are maximizing safety, equity, and convenience and minimizing time and cost. Each of these objectives was operationalized with a measureable attribute. Cost is defined as the screening fee that passengers pay per flight. Wait time is defined as the length of time in minutes that passengers, on average, had to wait to complete security screening. Convenience is defined as the proportion of passengers without contraband mistakenly singled out for further scrutiny. These false alarms are resolved by having these (misclassified) passengers undergo a more thorough screening, i.e. they have to take off their shoes, remove their jackets, remove their laptops, etc. Safety is defined as the miss rate, the proportion of passengers with contraband boarding a plane without being detected during screening. The "safety" attribute, as defined here, is probabilistically related to the notion of "safety" often used in the colloquial sense. The motivation for this definition stems from the fact that the higher the number of people who are carrying contraband on board, the higher the probability that passengers experience catastrophic security incidents. Equal protection is operationalized as the level of equity in the screening process. Specifically, the low equity policies alternative is a two-stage screening in which all travelers undergo a quick screening but some of them will be selected for an additional more thorough screening. The high equity alternative screening policy alternative involves a one-stage procedure that has all passengers undergo the same thorough security screening. The two-stage screening is considered less equitable than the one-stage screening.

\subsection{Trade-off Elicitation}

We applied an indifference procedure to quantify the values at which respondents were willing to sacrifice for safety. Respondents were asked to choose their preferred airline from a series of binary choices. The general approach was to discover a pair of screening alternatives varying on two attributes that respondents were indifferent. This assessment allowed for calculation of safety premiums and 
Table 1. Panel characteristics

\begin{tabular}{|c|c|c|c|c|c|c|c|c|}
\hline & & Baseline $(n=222)$ & Time $1(n=168)$ & Time $2(n=176)$ & Time $3 \mathrm{n}(170)$ & Time $4(n=166)$ & Time $5(n=172)$ & Time $6(n=174)$ \\
\hline \multicolumn{9}{|l|}{ Sex } \\
\hline & female & $47.748 \%$ & $63.095 \%$ & $60.227 \%$ & $62.353 \%$ & $63.855 \%$ & $61.628 \%$ & $60.920 \%$ \\
\hline \multicolumn{9}{|c|}{ Ethnicity } \\
\hline & African Americans & $6.306 \%$ & $4.762 \%$ & $7.386 \%$ & $7.059 \%$ & $9.036 \%$ & $4.651 \%$ & $6.322 \%$ \\
\hline & Asian Americans & $7.658 \%$ & $7.143 \%$ & $4.545 \%$ & $3.529 \%$ & $4.819 \%$ & $4.070 \%$ & $3.448 \%$ \\
\hline & Hispanic Orignis & $4.054 \%$ & $4.167 \%$ & $7.386 \%$ & $7.647 \%$ & $4.217 \%$ & $3.488 \%$ & $5.172 \%$ \\
\hline & Native Americans & $0.000 \%$ & $0.000 \%$ & $0.568 \%$ & $0.588 \%$ & $0.000 \%$ & $1.163 \%$ & $0.000 \%$ \\
\hline & Other & $0.901 \%$ & $0.595 \%$ & $2.841 \%$ & $4.118 \%$ & $0.602 \%$ & $2.326 \%$ & $1.149 \%$ \\
\hline & Pacific Islanders & $0.000 \%$ & $0.000 \%$ & $0.000 \%$ & $0.000 \%$ & $0.000 \%$ & $0.581 \%$ & $0.575 \%$ \\
\hline & Caucasians & $81.081 \%$ & $83.333 \%$ & $77.273 \%$ & $77.059 \%$ & $81.325 \%$ & $83.721 \%$ & $83.333 \%$ \\
\hline \multicolumn{9}{|c|}{ \# Fights last 4} \\
\hline years & None & $14.865 \%$ & $10.119 \%$ & $13.068 \%$ & $11.765 \%$ & $6.024 \%$ & $7.558 \%$ & $9.770 \%$ \\
\hline & 1 to 3 times & $51.802 \%$ & $44.048 \%$ & $46.591 \%$ & $47.059 \%$ & $42.771 \%$ & $52.907 \%$ & $39.655 \%$ \\
\hline & 4 to 6 times & $20.721 \%$ & $21.429 \%$ & $23.864 \%$ & $28.824 \%$ & $28.313 \%$ & $23.837 \%$ & $32.184 \%$ \\
\hline & More than 6 & $12.613 \%$ & $24.405 \%$ & $16.477 \%$ & $12.353 \%$ & $22.892 \%$ & $15.698 \%$ & $18.391 \%$ \\
\hline
\end{tabular}

relative weights for the MAU model. We illustrate the indifference procedure in Figure 2. The horizontal axis represents the two fixed levels of safety whereas the vertical axis represents levels of cost. A lower level of cost and higher level of safety are preferred. The letters represent the set of binary choices in three trials in which choice $\mathrm{A}$ is fixed and choice B is varied. In this graphical presentation, we consider the trade-off between safety and cost represented in a series of three binary choice trials with two options, A and B. The level of safety for A is low, and the cost associated with this screening is also low. On the other hand, option B offers a more expensive screening cost but the level of safety is higher.

Respondents were asked to choose either A or B1 in the first trial. The choice of option B1 suggests people prefer safety over cost in this particular tradeoff, whereas the choice of option A suggests otherwise. Since our goal was to elicit respondents' indifference point, the choice of B1 implies that the cost associated with this option should be higher, and the choice of A implies that the cost of B1 should be lower. Thus, the cost in option B1 was adjusted to B2 $>\mathrm{B} 1$ if respondents chose $\mathrm{B} 1$, or $\mathrm{B} 3<\mathrm{B} 1$ if $\mathrm{A}$ was chosen assuming the cost for $\mathrm{A}$ was fixed. The same logic was applied in the next trial, which resulted in four possible positions for option $\mathrm{B}$. The procedure was repeated until respondents indicated they were indifferent between the two options. The difference in cost between A and B in the indifference trial is the safety premium. This value is interpreted as the premium that people are willing to sacrifice for safety.

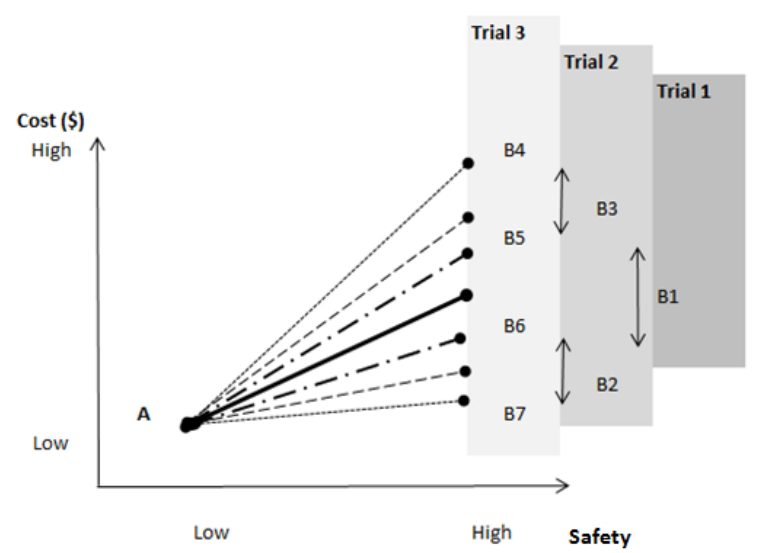

Figure 2. Indifference methodology

If a respondent did not select the "indifference" option in any of the three trials, their safety premium was bounded using inequalities determined from the three trials. We set the midpoint value of the bounded range as the safety premium for respondents who did not indicate indifference at any three trials. For respondents who consistently chose the higher cost option for all 3 choices, we set the safety premium to be twice as large as the cost in the third trial. Other pairs of trade-offs followed the same logic. 


\subsection{Screening Procedure Manipulation}

To examine the effect of alternative screening procedures on equity premiums (and relative weights), we manipulated the selection procedure in the two-stage screening for choice tasks involving variation in equity. Specifically, the one-stage screening requires that every air traveler is screened in the same manner. The two-stage screening requires an initial screening of all travelers (less intrusive than the one-stage screening). However, certain passengers are selected for additional, more thorough screening. The two-stage screening is presumed to be less equitable than the one-stage screening. The focus of the manipulation is how the passengers are selected for additional scrutiny in the two-stage screening. Air travelers are selected as a result of one of the following procedures: random process, behavioral selection, or demographic profiling. Randomized selection is defined as the random selection of passengers based on a computer algorithm. Behavioral selection uses trained TSA officers to identify passengers based on suspicious behavioral indicators, such as perceived fear or stress. Demographic profiling selects passengers based on their age, race, sex, and/or national origin.

\section{Results}

We first compute weights for each of the five objectives by assuming linear utility functions for all 5 attributes. Although we elicited weight ratios for all 10 attribute pairs, any four ratios involving all 5 attributes would yield an estimate of the scaling parameters (relative weights) for all five attributes. We chose to use the four assessments involving per flight cost to compute relative weights, consistent with the "pricing out" method by Keeney and others [17]. Examinations of the distributions revealed nonnormality, suggesting a violation of one of the assumptions in parametric statistics. Thus, we conducted all of the analyses using non-parametric statistics. First, Kruskal-Wallis, a non-parametric version of one-way ANOVA, was used to detect changes in distributions of weights over time. Follow-up Kolmogorov-Smirnov (KS) tests, a nonparametric version of t-test, were used to compare different pairs of time points. Second, the same tests were used to compare the effects of the experimental groups. In the next sections, we present descriptive results graphically, followed by statistical tests.

\subsection{The Effect of MH370 on Preference for Safety}

Figure 3 presents the cumulative distributions of weights for each of the five attributes over time. In each of the five plots, each curve represents the distribution of weight at a particular point in time when the data were collected. The horizontal axis in each plot represents the value of weights for a particular objective while the vertical axis is the cumulative percentage. The curves to the lower right (following disappearance of MH370) do not cross the curves to the upper left (prior to the disappearance of MH370). Consequently, safety was judged as being relatively more important following loss of MH370 compared to the baseline obtained prior to the MH370 loss. Stated differently, respondents following loss of MH370 (lower curves), were willing to make greater sacrifices for safety, i.e. a greater safety premium, than respondents prior to the MH370 disappearance (higher curves). Visual inspection suggests a clear effect of the disappearance of the MH370. In particular, safety has received more weight after the disappearance. Unexpectedly, weight for convenience also increased after the disappearance of MH370. On the other hand, relative weights for time, money, and equity all decreased. Interestingly, the shifts in weight distributions appear from the very first assessment following the MH370 disappearance (time 1). In fact, there is little variation among the distributions for times 1 through 6 , as indicated by the similar cumulative plots.

Statistical tests confirmed these visual inspections. Kruskal-Wallis (KW) test suggested a significant difference among the distributions over time across all five objectives: for safety, $\mathrm{KW} \chi^{2}(6)$ $=84.582, p<. .001$; for equity, $\mathrm{KW} \chi^{2}(6)=440.607$, $p<$. .001; for convenience, $\mathrm{KW} \chi^{2}(6)=442.44, p<$. .001 ; for time, KW $\chi^{2}(6)=121.293, p<. .001$; and for money, KW $\chi^{2}(6)=442.80, p<. .001$ Followed up pairwise comparisons with KS tests and Bonferroni correction for type I error suggested the significant differences between the baseline and each of the time point when data were collected after the MH370 disappearance, $p s<.01$.Comparisons among other time points revealed no significant difference. 


\subsection{The Effect of Alternative Screening Procedures on Preference for Equity}

Our analysis of the effect of secondary screening selection procedures was carried out on the combined data from the six time periods following the disappearance of MH370 only.
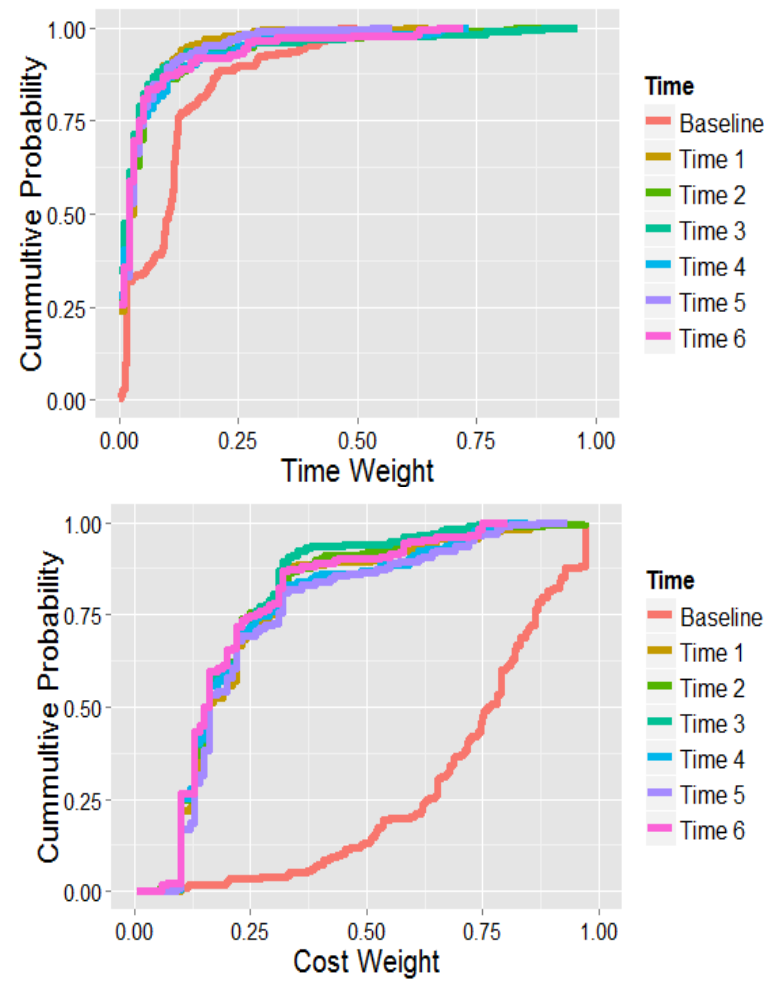

- Baseline

- Time 1

- Time 2

- Time 3

- Time 4

- Time 5

- Time 6

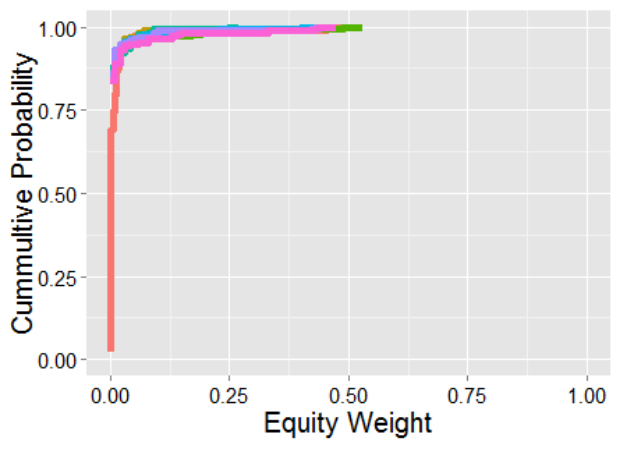

Figure 3. Cumulative distributions of weights

Equity received higher relative weight in the profiling condition and lower relative weight in the randomization condition. Follow-up KS tests confirmed this observation. There were significant difference in the distribution of equity weights between the profiling and the randomization conditions, $D=0.095, p=.046$. The difference between the behavioral screening and randomization was in the expected direction, but not significant, $D=$ $0.090, p=.059$. There was clearly no significant difference between the profiling and the behavioral selection conditions.
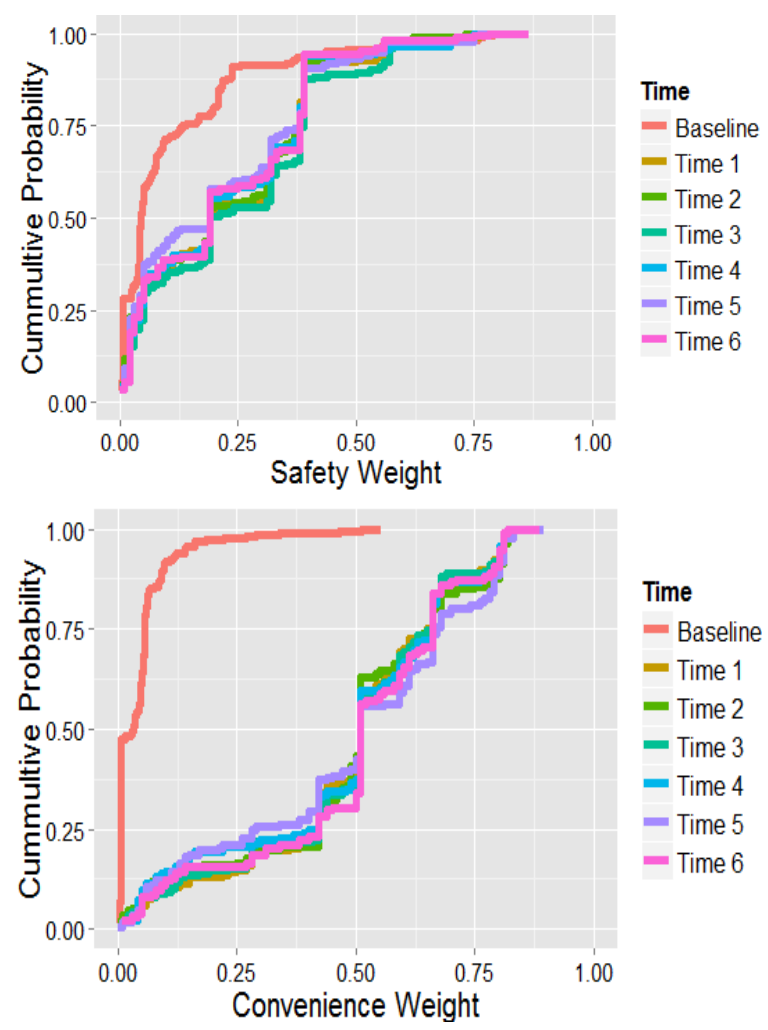

Figure 3 (cont)

\section{Discussion}

Utilizing the Malaysian Flight MH370 incident, this research examines changes in safety trade-offs. Using multi-attribute utility theory, we conceptualized and elicited trade-offs for safety within a broad value system involving five multiple and conflicting objectives, and modeled how the trade-off for safety changed as a result of the MH370 incident. While the time decay hypothesis suggests a gradual attenuation of safety weight as the event fades, our data does not provide empirical support for this prediction. In fact, the results suggest a relatively enduring effect of the incident on safety concerns. Indeed, the weight for safety was relatively constant over a period of six weeks after the incident had occurred. Importantly, the values of safety elicited after the incident were significantly larger than the baseline value measured before the incident, suggesting that air travelers were willing to make greater sacrifices for safety after the event. As 
expected, weights for (some) other objectives decreased as the relative weight for safety increased, lending further support for the finding. To our awareness, this is one of the first (and few) studies that tracks and measures changes in trade-offs for safety over time, and examines the effect of a realworld event on travelers' trade-offs.

The finding that safety concerns were relatively constant over an extended period of time (following disappearance of MH370) was surprising, given the robust empirical support for the time decay hypothesis in the literature on traffic accident [7]. Nevertheless, evidence against the time decay hypothesis has also been reported in other studies. For example, Wernstedt and Murray-Tuite ${ }^{[12]}$ used survey data to model trade-offs for safety after the collision of two DC Metrorail's trains. Researchers found a significant and positive relationship between trade-offs for safety and time, which suggests that passengers valued safety more as the event faded. However, the lack of a baseline measure limited their conclusions. In contrast, we utilized a baseline measure in our experiment, which made it experimentally feasible to examine the effect of an ambiguous incident on trade-offs involving safety. Secondly, whereas researchers in the previous study used statistical models to infer safety weights, we elicited safety trade-offs in this study. Thus, relative safety weights, as elicited in this study, are a reflection of respondents' subjective judgments rather than statistical parameters.

Importantly, whereas the previous study measured preference (or concern) for safety from respondents who were at least indirectly affected by the collision, we obtained similar results by recruiting respondents who were not affected by the incident. When there are immediate safety risks, it is perhaps not surprising that people are willing to make greater sacrifices for safety due to the objective risk. However, we found that when safety risks are not immediate (indeed they were quite remote), people are also willing to make greater trade-offs for safety, suggesting the importance of risk perception in behavioral responses after extreme disaster incidents.

Similar to an increase in relative safety weight, respondents also indicated a greater weight for convenience after the incident. One possible interpretation for the increasing importance of convenience is changes in airport security procedures following the MH370 incident, which possibly elevated the level of inconvenience experienced by travelers. It follows that respondents would thus put a greater premium on convenience following the incident. While there was little actual change in security procedures immediately following the loss of
MH370, it is possible that travelers expected an increase in inconvenience after the incident. An alternative, and perhaps more plausible account involves respondents' expectancy for more security inconvenience. Recall that terrorism was not ruled out as one of the possible causes of the incident during the study period. Thus, it is reasonable to believe that respondents may have expected more airport security hassles, and this could account for an increased weight for convenience following the loss of MH370. Certainly, this finding and interpretation should be explored further.

Because of the premium placed on equity, several airport security measures have been delayed or discontinued. Understanding travelers' concern for equity in the context of airport security is important. We found that different risk-based screening procedures produce different effects on the relative weight for equity. The notion of procedural fairness implies that the perceptions of the (security) procedure that legal authorities (e.g., the TSA) apply are more important than the outcome of the procedure. Thus, by controlling for the effectiveness and outcome of the selective security screening, we focused on the procedural characteristics of selecting passengers for further security checks. We found that air travelers were willing to make greater sacrifices to avoid a procedure that selects passengers based on their demographic profiles compared to procedures that single out travelers based on their behavioral expression characteristics or a randomized process. These results offer valuable insights regarding the use of different airport security screening procedures. For instance, even if the use of profiling results in greater effectiveness compared to behavioral selection and/or randomization, this benefit must be weighed against the risk of public rejection due to concerns about equal protection.

Most decisions involve multiple objectives, and these objectives are often conflicting, requiring decision makers to make difficult trade-offs. The decision to select and adopt airport security policy to make air traveler safer is not an exception. The TSA, airport authorities, and commercial airlines need to consider how various characteristics of a proposed policy may hinder or promote values about which air travelers are deeply concerned. Importantly, understanding how travelers make trade-offs for various policy attributes will provide critical insights for those stakeholders to consider and (re)design proposed security initiatives in a manner that the traveling public will find useful, effective, and acceptable. The current study makes this approach feasible by 1) providing new insights on how travelers value safety when there is an aviation safety 
incident and 2) suggesting that preference for equity, which is inversely related to safety, is contingent upon the risk-based screening measure used.

Ultimately, the success of a particular security policy depends on how it is perceived with respect to public concerns and values that may not be directly related to the effectiveness or efficiency of the policy under consideration. Assessing public trade-offs related to deeply held personal values such as safety and equal protection (equity), therefore, is a potentially useful tool to better understand the complexity of public values essential to formulating sensible and effective security policies.

\section{References}

[1] K. Frankovic. (2015, April 1). "Most U.S.s now worried about flying". Economist/YouGov Poll Life. https://today.yougov.com/news/2015/04/01/most-U.S.snow-worried-about-flying/. Accessed 27 August $206 \backslash 16$

[2] DW. Davis, and BD. Silver. "Civil liberties vs. security: Public opinion in the context of the terrorist attacks on America. U.S. Journal of Political Science", 2004; 48(1): 28.

[3] E. O'Keefe, and A. Halsey III. (2011, September 6). "Shoe removal requirement at airports to be phased out". The Washington Post. http://www.washingtonpost.com/ local/shoe-removal-requirement-at-airports-to-be-phasedout/2011/09/06/gIQAknLD7J_story.html. Accessed 27 August 2016

[4] RL. Keeney, and H. Raiffa. Decisions with multiple objectives: trade-offs and value tradeoffs. New York, USA: Cambridge University Press. 1993

[5] WK. Viscusi, and RJ. Zeckhauser. "Sacrificing civil liberties to reduce terrorism risks". Journal of Risk and Uncertainty, 2003; 26: 99-120

[6] WK. Viscusi, and RJ. Zeckhauser. "Recollection bias and the combat of terrorism". The Journal of Legal Studies, 2005; 34(1): 27-55.

[7] T-C. Lirn, and JB. Sheu. "The impacts of an air-crash on students' transportation choice behaviour: An empirical study undertaken in Taiwan". Transportation Research Part F: Psychology and Behaviour, 2009; 12(5):404-416.
[8] P. Dixon, G. Rehling, and R. Shiwach. "Peripheral victims of the herald of free enterprise disaster". British Journal of Medical Psychology, 1993; 66(2):193-202.

[9] F. Prager, B. Fasolo, and Z. Ni. "Analysis of passengers' reactions to the sarin gas attacks in Tokyo". In: D. Winterfeldt, and P. Fynnwin. (Eds). Estimating Behavioral Changes for Transportation Modes after Terrorist Attacks in London, Madrid, and Tokyo. Los Angeles, CA: METRANS Transportation Center; 2010. p. 81-90.

[10] T. Baumert. "The impact of the 3/11 Madrid bombings on consumers travel behavior". In: D. Winterfeldt, and P. Fynnwin. (Eds). Estimating Behavioral Changes for Transportation Modes after Terrorist Attacks in London, Madrid, and Tokyo. Los Angeles, CA: METRANS Transportation Center; 2010. p. 60-79.

[11] F. Prager, G. Asay, B. Lee, and D. von Winterfeldt. "Exploring reductions in London underground passenger journeys following the July 2005 bombings". Risk Analysis, 2011; 31(5): 773-786.

[12] K. Wernstedt, \& P. Murray-Tuite. (2014). "The Dynamic Nature of Risk Perceptions After a Fatal Transit Accident". Risk Analysis, 35(3), 536-552. doi:10.1111/risa. 12284

[13] National Research Council. Fairness and effectiveness in policing: The evidence. In: W. Skogan, and K. Frydl. Committee on law and justice, division of behavioral and social sciences and education. Washington, DC: The National Academies Press; 2004.

[14] M. Buhrmester, T. Kwang, and SD. Gosling. "Amazon's Mechanical Turk: A new source of inexpensive, yet high-quality, data?" Perspectives on Psychological Science, 2011; 6(1): 3-5.

[15] W. Mason W, and S. Suri. "Conducting behavioral research on Amazon's Mechanical Turk". Behavior Research Methods, 2012; 44(1): 1-23.

[16] G. Paolacci, J. Chandler, and PG. Ipeirotis. "Running experiments on Amazon Mechanical Turk". Judgment and Decision Making, 2010; 5(5): 411-419

[17] RT. Clemen. "Making Hard Decisions: An Introduction to Decision Analysis". PWS-Kent

Publishing Co., Boston, MA. 1996 